\title{
My 50-Year Journey from Punched Cards to Swarm Systems
}

\author{
Alberto Sangiovanni Vincentelli \\ Department of EECS, University of California \\ Berkeley, California, USA \\ alberto@berkeley.edu
}

\section{EXTENDED ABSTRACT}

The article is a reflection on my journey during the development of the EDA field, from its early days to its explosive growth and present maturity. The two special issues of the Solid State Circuit Society Magazine "Corsi e Ricorsi: Alberto Sangiovanni Vincentelli and the Evolution of EDA", published in 2010 [1, 2], contain a set of papers that pinpoint some of the stages of this journey.

When I graduated from College in 1971, integrated circuits were in their infancy: few tens of transistors were the norm. Masks were prepared by hand on rubylith with pain and fear to make mistakes. Computers were large mainframes but paling with respect to the computing power that we have available today. There were no graphic terminals and the common ways to provide inputs to a computer were paper tapes and punched cards. Not really an exciting field to invest my future in (or at least so it seemed!). I have always been attracted by algorithms and mathematical formalism, thus I began my research work on system theory with particular attention to large-scale systems. When I went to Berkeley in 1975, I began my work in EDA with numerical analysis methods for solving large scale systems of ODEs. At that time, I joined the Berkeley integrated circuit simulation effort by developing some of the numerical algorithms embedded in the most used academic program in the world, SPICE. My students (E. Lelarasmee and J. White) developed a new set of relaxation-based algorithms (waveform relaxation) for ODEs that not only influenced generations of fast circuit solvers still in use today but also created a new numerical analysis area called dynamic iteration.

In the early 1980s, spurred by my collaboration with IBM, I expanded my research interests to automatic IC layout and logic synthesis. While spending a sabbatical at the IBM Yorktown Research Center, I saw the power of the simulated annealing approach to optimization as proposed by Kirkpatrick. My students and I studied a mathematical analysis of the convergence properties of the algorithm. Based on these findings, we developed automatic layout algorithms embodied in Timber Wolf, authored by C. Sechen, YACR (Yet Another Channel Router) and Mosaico

Permission to make digital or hard copies of part or all of this work for personal or classroom use is granted without fee provided that copies are not made or distributed for profit or commercial advantage and that copies bear this notice and the full citation on the first page. Copyrights for third-party components of this work must be honored. For all other uses, contact the Owner/Author. ISPD '19, April 14-17, 2019, San Francisco, CA, USA.

(C) 2019 Copyright is held by the owner/author(s).

ACM ISBN 978-1-4503-6253-5/19/04.

DOI: https://doi.org/10.1145/3299902.3311071 that were extensively used in industry (Intel, DEC, TI, National Instruments, Motorola, AMD, and ST Microelectronics, all used the layout tools we developed). Contemporary to this work, my collaborators, my students and I developed a suite of algorithms and programs for two-level (ESPRESSO) and multi-level (MIS) logic synthesis. Logic synthesis was a breakthrough technology as it allowed transforming a Register-Transfer Level description into a set of optimized logic gates by using mathematical techniques. These techniques are still the ones in use today.

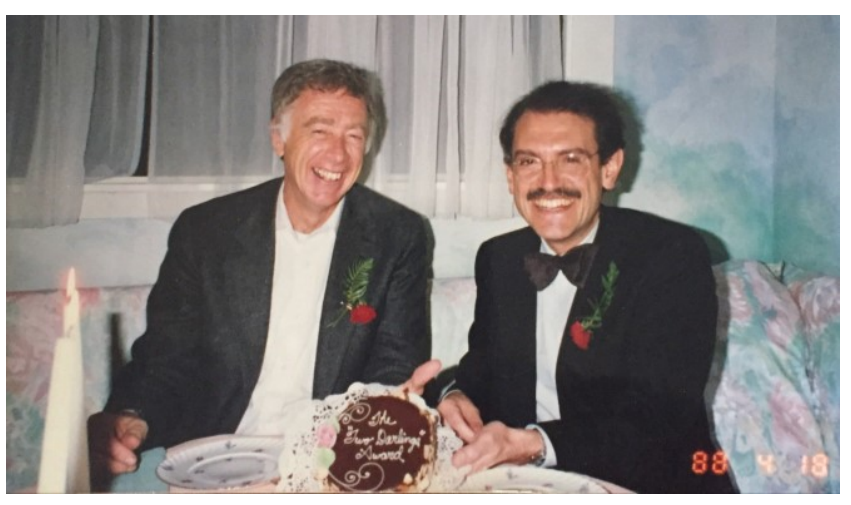

Figure 1: Robert Bryton and Alberto Sangiovanni Vincentelli at the Darlington Award ceremony (1988).

By 1982, it was clear that the body of knowledge assembled by the Berkeley group was having a very strong impact in industry worldwide. Pressure mounted to form a new EDA company that could bring this technology to market in a robust and sustainable way. Together with colleagues Newton and Solomon, I founded SDA, which later became Cadence. IC design was largely carried out with few expensive tools running on large mainframes or specialized graphic processors. The design process was slow, often manual and error prone for the lack of verification and analysis tools. The electronic industry was confronted with serious productivity problems. We changed the situation: the introduction of layout editing and simulation on inexpensive general-purpose workstations as well as the introduction of algorithms for the automatic layout of gate-array and standard-cell chips yielded at least one order of magnitude productivity improvements. It made it possible to develop semiconductor chips that were working correctly the first time as opposed to the several manufacturing runs needed earlier. SDA was the first software-only company: other EDA companies in existence at that time were offering a mix of hardware and software solutions. The focus on software only 
solutions helped providing margins that propelled the company to be one of the 10 largest software companies in the world today.

Parallel to the foundation of SDA, I was interacting closely with most of the leading semiconductor operations in the world: IBM, Intel, Bell Labs, Texas Instruments, HP, ST Microelectronics, Hitachi, Fujitsu, Philips as advisor for design methods and tools. In 1984, the Intel 386 design team headed by Gelsinger decided to rely upon the entire suite of tools we had developed so far. The success of the design and of the tools was a landmark in University-Industry collaboration. The DEC Alpha processor was developed using our design tools in an unprecedented effort to reduce power consumption without losing performance.

After the Berkeley logic synthesis tools became widely used, Aart de Geus and a group of North Carolina GE developers teamed with Richard Newton and me to form Synopsys. At that time, I was instrumental in evangelizing the shift from a channels-and-rows based semi-custom architecture to the sea-of-gates/sea-of-cells architecture. My PhD students (R. Rudell and A. Wang) formed a core part of the product team for Design Compiler, the most successful single product in the history of the EDA industry to date, which was heavily influenced by our results and, in particular, by the contributions of Robert Brayton.

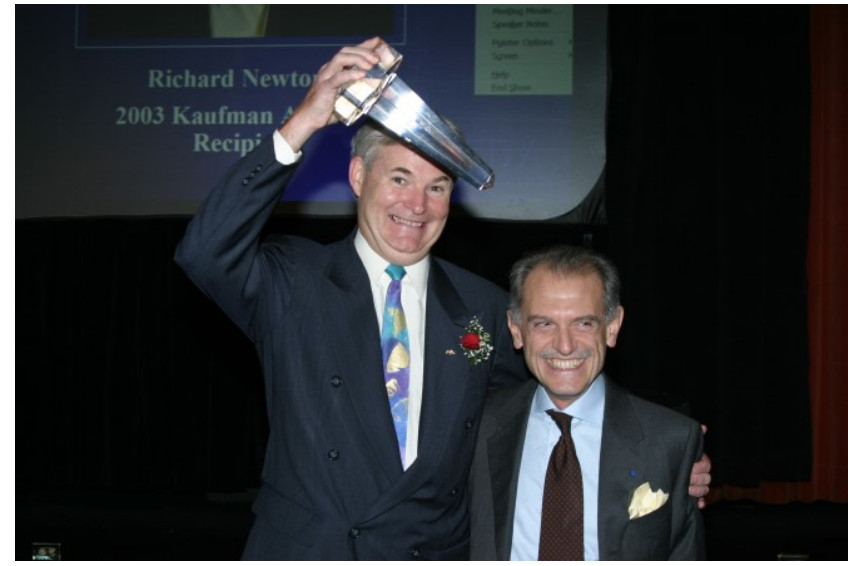

Figure 2: Richard Newton and Alberto Sangiovanni Vincentelli celebrating Richard's Kaufman Award (2003).

By 1992, Cadence and Synopsys were the two leading companies in EDA. Both were public companies and started expanding into each other's markets. Because of conflict of interest, I had to make a hard choice between the two companies. Since 1992, I played a fundamental role in Cadence as a Board Member, Chief Consultant first and then Chief Technology Advisor by helping to guide the technology development process in the company and helping to form its strategy. In 2001, Cadence CEO Bingham and I received in the name of Cadence the IEEE Corporate Innovation Recognition Award.

In the 1990s, I focused my attention to system design and, in particular, to the automotive field. During that period, I started my collaboration with Magneti Marelli and Daniele Pecchini who allowed me to test the ideas and to develop them further in an industrial environment. During this period, I also worked with Mercedes and BMW to help with the development of methodologies and tools for automotive design. The result of this collaboration is the development, in collaboration with Alberto Ferrari, of the foundations of Platform-based Design (PBD), a novel approach to system design. In 1998, the Gigascale System Research Center (GSRC) was established in Berkeley. PBD was a major result of that research program where I led the system-level design group. Because of my interest in the foundations of the design process, PBD was developed to cover the design process for complete systems as large as an automobile or an airplane as well as for subsystems such as a chip or an Electronic Control Unit. The method leverages mathematical concepts that allow assembling designs with heterogeneous components and verifying their properties. Magneti Marelli, a European Tier-1 supplier of automotive electronics, deployed broadly the methodology during the period 1995-1998, obtaining major results in productivity and quality. UTC has recently mounted a major program to introduce a version of the methodology across its various businesses with the efforts of Alberto Ferrari. These efforts continued with Musyc and then with Terra Swarm, two MARCO programs supported by DARPA and the Semiconductor Industry Association, where Cyber-Physical Systems (CPS) and Swarm Systems where the main focus.

In 2006, following the development of work on hybrid and embedded systems with Marika Di Benedetto, Edward Lee and Shankar Sastry, a group was formed to push for a major research effort on systems where interactions between interconnected computing systems and the physical world are of primary importance. This push yielded the manifesto for cyber-physical systems that started a major research and development effort worldwide. Industry 4.0 , as articulated by a 2011 acatech (the German Academy of Science and Technology) report, is based on CPS. The manifesto was the basis for an NSF program that started in 2011 and it is still going today.

In parallel, given my European interests and interactions, I contributed to the formation of the European Community Artemis Joint Technology Initiative, a Public Private Partnership that involves most of the European system and electronics companies. Several EU projects in collaboration with A. Benveniste, P. Caspi, W. Damm and J. Sifakis investigated theory and implementation issues in CPS. With M. Di Natale and students, and in particular, with L. Carloni, A. Pinto, C. Pinello, Q. Zhu and A. Davare, we aimed at defining new architectures for chips and systems where guarantees on timing and functionality could be rigorously assessed.

With the strong interest on synthetic biology brewing at Berkeley, D. Densmore developed a set of tools based on PBD, to design new form of life from bio-bricks, an effort that Densmore is still pursuing in his academic career and start-up efforts. An additional application of PBD is in energy efficient buildings. The Singapore Berkeley SinBerBEST effort has been the home of this research that started in collaboration with UTC, students Q. Zhu, Y. Yang and M. Maasoumy, and C. Spanos. 
PBD took an interesting turn when it was married with Contractbased Design developed once more in collaboration with A. Benveniste, W. Damm, R. Passerone and others. In particular, the application of PBD to the design of aircraft power distribution systems married with contracts yielded an interesting set of tools and methodologies in collaboration with R. Murray and with the substantial contribution of P. Nuzzo. Contract-based design is now an important effort that is pursued in several academic and industrial circles. Given the growing interests in security, we are presently pursuing with S. Seshia the application of contracts to secure hardware and software systems including swarm systems such as autonomous vehicles.

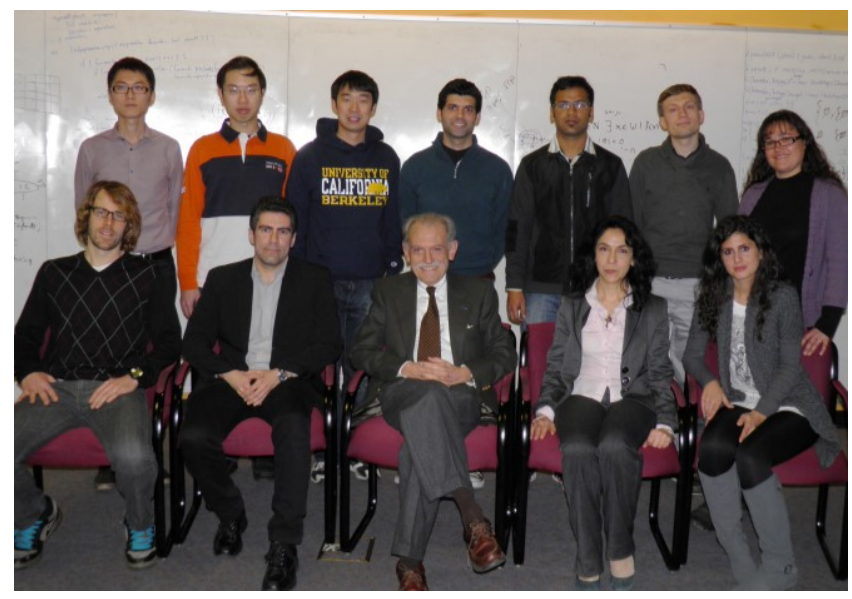

Figure 3: Alberto's group picture in the Donald O. Pederson Center for Electronic Systems Design at Berkeley (2014).

In summary, my journey has been a continuous search for new avenues where theory can be married to applications and yield provable improvements in all aspects of design.

\section{CCS CONCEPTS}

- Design • Verification • Design reuse and communicationbased design • Platform-based design • Electronic design automation (EDA) • Logic synthesis • Physical design • Methodologies for EDA • Embedded software

\section{KEYWORDS}

Electronic Design Automation; System Level Design

\section{BIOGRAPHY}

Alberto Sangiovanni Vincentelli has served on the faculty of the University of California, Berkeley since 1976 and currently holds the Edgar L. and Harold H. Buttner Chair of Electrical Engineering and Computer Sciences at the University of California at Berkeley. As both an academic and entrepreneur, Alberto Sangiovanni Vincentelli propelled electronic design automation (EDA) into an indispensable engineering discipline with his scientific contributions, collaborations with industry and by cofounding the two largest EDA companies (Cadence and Synopsys) in the world. During the 1990s, Sangiovanni Vincentelli developed the foundations of "platform-based design," a comprehensive

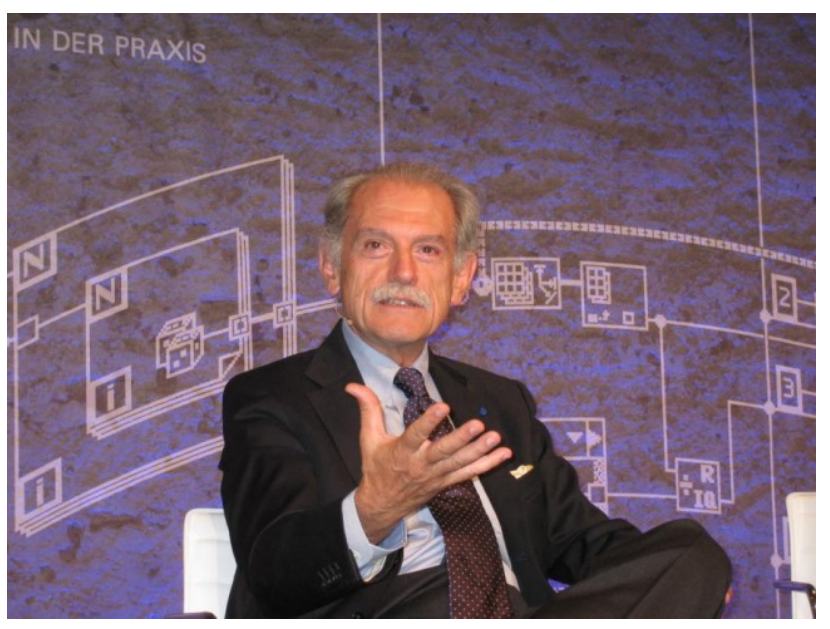

design and analysis methodology for electronic systems. In 2001, he received the Kaufman Award for his pioneering contributions to EDA from the Electronic Design Automation Consortium. In 2011, he was awarded the IEEE/RSE Maxwell Medal "for groundbreaking contributions that have had an exceptional impact on the development of electronics and electrical engineering or related fields". He received an honorary Doctorate from Aalborg University (Denmark) and one from KTH (Sweden). Dr. Sangiovanni Vincentelli presently serves on the Board of Directors of Cadence Design Systems Inc., KPIT Technologies, Sonics, Cogisen, Expert System, UltraSoC (Chairman of the Board), and on the Advisory Board of Atlante Ventures, XSeed, and Walden International. An advisor to leading companies such as Elettronica, Camozzi Group, LendLease, Intel, HP, TI, ST Microelectronics, Mercedes, BMW, Magneti Marelli, Telecom Italia, United Technologies, General Motors and Pirelli, he served as the President of the "Comitato Garanti per la Ricerca" of the Italian Government, the Chairman of the Strategy Committee of Fondo Strategico Italiano and is serving as member of the Advisory Board of the Politecnico di Milano, and as Chairman of the Advisory Board of the MIND (Milano Innovation District) Program. He is a member of the National Academy of Engineering, an IEEE and ACM Fellow. He has published more than 950 papers and 17 books in the areas of EDA, design methodologies, control, hybrid systems and system-level design.

\section{ACKNOWLEDGMENTS}

My contributions would not have been possible without the support and collaboration of my dearest colleagues and friends, Bob Brayton and Richard Newton, as well as my graduate students and co-workers. I want to acknowledge the support I received over the years from DARPA, the National Science Foundation, the European Community and industry.

\section{REFERENCES}

[1] Corsi e Ricorsi: Alberto Sangiovanni Vincentelli and the Evolution of EDA, IEEE Solid State Circuit Society Magazine, vol. 2, n. 3, Summer 2010

[2] Corsi e Ricorsi: Alberto Sangiovanni Vincentelli and the Evolution of EDA, IEEE Solid State Circuit Society Magazine, vol. 2, n. 4, Winter 2010 